\title{
Changes in the Temporal Structure of Periodic Breathing with Postnatal Development in Preterm Infants
}

\author{
M. H. WILKINSON, S. CRANAGE, P. J. BERGER, N. BLANCH, AND T. M. ADAMSON \\ Institute of Reproduction and Development [M.H.W., P.J.B., N.B.] and Department of Pediatrics [S.C., \\ T.M.A.J, Monash Medical Centre, Clayton, Victoria, 3168, Australia
}

\begin{abstract}
The progressive decrease in the periodic cycle duration (PCD) of periodic breathing with postnatal age in term infants has been previously reported by a number of authors and is thought to be associated with peripheral chemoreceptor maturation. We hypothesized that a similar decrease should be observed in preterm infants. Therefore, in this study we measured the changes in PCD with postnatal age in a small group of preterm $(n=4)$ infants followed longitudinally (36 afternoon nap studies) over the first 6 mo postnatally. PCD declined in these infants from $17.1 \pm 3.3 \mathrm{~s}($ mean $\pm 2 \mathrm{SD}$ ) at $9 \mathrm{~d}$ to $9.8 \pm 3.2 \mathrm{~s}$ (mean \pm $2 \mathrm{SD})$ at $105 \mathrm{~d}$. The regression slope was $-0.072 \mathrm{~s} / \mathrm{d}$. Beyond $105 \mathrm{~d}$ there was no change in PCD up to 6 mo postnatally. We found no significant difference between active and quiet sleep. These results are similar to results previously published in term infants but apparently contradict recent data on a group of preterm infants. Possible reasons for this discrepancy are discussed. By examining long epochs of periodic breathing in these infants we also identified characteristic changes in PCD and V/A ratio, defined as the duration of the ventilatory period divided by the duration of the apneic interval. V/A ratio fell from the start of an epoch from $1.21 \pm 0.08$ (mean $\pm S E M$ ) to a minimum of 0.62 \pm 0.03 and then increased again to $0.8 \pm 0.05$ at the end of the epoch. We conclude that important maturational changes occur
\end{abstract}

Periodic breathing has been studied extensively in both preterm and term infants. These studies have been aimed primarily at elucidating the causes of this type of breathing pattern in neonates and establishing the incidence during the first months of life $(1,2)$. Much of the incentive for these studies came from earlier clinical observations that long apneas were often preceded by periodic breathing in preterm infants (3), although this finding has been disputed more recently (4). Also, a high incidence of periodic breathing has been reported in apparent life-threatening events and SIDS sibling infants when compared with age-matched con-

Received August 3, 1994; accepted May 18, 1995.

Correspondence: Dr. M. H. Wilkinson, Institute of Reproduction and Development, Monash Medical Centre, 246 Clayton Road, Clayton, 3168 Australia.

This work was supported by grants from the National Health and Medical Research Council of Australia and the National SIDS Council of Australia. in the neonatal respiratory control system during the first 6 mo postnatally and that these changes are reflected in a fall in PCD of periodic breathing over this period. We also conclude that the characteristic changes which occur in V/A ratio and PCD are consistent with a role for chemical stimuli in the genesis of periodic breathing. (Pediatr Res 38: 533-538, 1995)
SIDS, sudden infant death syndrome
V/A, ratio of the duration of ventilation to the duration of apnea in one cycle of periodic breathing
$T_{o}$, start time of a long epoch of periodic breathing
$\boldsymbol{T}_{\min }$, time from To at which V/A ratio reaches its minimum value
$T_{\text {end }}$, end time of a long epoch of periodic breathing
PCD, periodic breathing cycle duration
$\triangle$ PCD, change in PCD during long epochs
PNA, postnatal age
PNA $\mathbf{A R}_{\mathbf{B R}}$, postnatal age at which PCD ceases to decrease
$\mathrm{SaO}_{2}$, arterial oxygen saturation
$\Delta \mathrm{SaO}_{2}$, deviation between the mean $\mathrm{SaO}_{2}$ and the peak or
trough of the oscillation in $\mathrm{SaO}_{2}$ during periodic breathing

trols, suggesting that this breathing pattern may be connected with some underlying maturational defect in respiratory control occurring within the first few months of life $(5,6)$.

Several studies have attempted to define the maturational changes in the temporal structure of periodic breathing in full-term infants during the first 6 mo of life (7-9). All of these studies demonstrated a characteristic fall in PCD over this period. Because periodic breathing is believed to be mediated through the action of cyclical hypoxemia and hypocapnia on the peripheral chemoreceptors (10), Wilkinson et al. (8), and Barrington et al. (9) suggested that the measured decrease in PCD might be useful as an index of the maturation of peripheral chemoreceptor function.

In light of these findings, we hypothesized that a similar change in PCD with postnatal age should occur in premature 
infants. Hence, we set out to gather data on the longitudinal maturation of PCD during the first six postnatal months in a group of preterm infants studied during an afternoon nap. To provide further insight into the causes of periodic breathing and to define sources of variability in PCD we also examined long epochs of periodic breathing to determine whether characteristic changes in PCD and V/A ratio occur during the course of these epochs. For comparison, we have included in this report relevant data values for term infants available from our previous study using an identical methodology (8). In this latter study, PCD and V/A ratio (during long epochs) were studied over the first 6 mo postnatally in four of seven healthy term infants.

Our results from this current study demonstrate that a significant decrease in mean PCD occurs over the first 6 mo postnatally in preterm infants. We also demonstrate that a characteristic decrease in V/A ratio and PCD occurs during the course of long epochs of periodic breathing. These maturational changes are similar to those observed previously in term infants and are consistent with a role for chemical stimuli in the genesis of periodic breathing.

\section{METHODS}

Study group. Five preterm infants entered the study, four female and one male. Their gestational ages and birth weights were: 27 wk (850 g), 29 wk (1170 g), 31 wk (1489 g), 32 wk $(1940 \mathrm{~g})$, and $34 \mathrm{wk}(2025 \mathrm{~g})$. Three infants were born by vaginal delivery and two by cesarean section. Apgar scores at 1 min ranged from 4 to 9 and, at $5 \mathrm{~min}, 8$ to 9 . One infant had hyaline membrane disease, requiring mechanical ventilation for $3 \mathrm{~d}$. Three infants had recurrent apnea treated with oral theophylline. In all infants apnea and theophylline had ceased by 34 wk of gestational age.

Before acceptance into the study a full examination was performed on all infants to exclude abnormalities and to ensure compliance with the longitudinal nature of the study. At each monitoring session, details of any recent infections, sleep pattern, and weight gain were recorded. The growth velocity in all instances over the study period was normal. Normal neurologic and developmental examination was confirmed by one of us (T.M.A) during and at the end of the study period, and at 12 mo of age (corrected for prematurity). The study was approved by the Queen Victoria Hospital Ethics Committee.

Monitoring period. The details of the recording and equipment have been described elsewhere (11). All recordings were performed by the same person (S.C.) in an air-conditioned room $\left(22-24^{\circ} \mathrm{C}\right)$ with controlled lighting, free from outside noise. Each monitoring period was performed during the afternoon between two feeds. Before the feeds the electrodes were attached to the infant who was then fed and placed in a cot.

The recording was started when the infant was settled and continued until the infant awoke, or until $2 \mathrm{~h}$ of recording had been obtained. It was planned for each infant to have nine recordings. Recordings were performed at 34,36 , and $38 \mathrm{wk}$ and term, and then a further five recordings were made at approximately 1 -mo intervals up to 6 mo postterm. These times were prearranged, and no recordings were canceled because of illness.

Recording equipment. Each infant had its respiratory patterns monitored by two nasal thermistors placed beneath the nares, and by a custom-designed abdominal movement transducer placed on the upper abdomen. The signals from these transducers were amplified, filtered and recorded on an eight channel thermal recorder (Hewlett Packard 7758B) at $2.5 \mathrm{~mm} / \mathrm{s}$ and on magnetic tape (Tandberg Series 100 or Hewlett Packard Instrumentation Tape Recorder $3968 \mathrm{~A}$ ) at $2.38 \mathrm{~cm} / \mathrm{s}$. This dual recording of breathing movements and airflow combined with visual observation permitted classification of apneas into central, obstructive, or mixed. In all infants (from $36 \mathrm{wk}$ ) EEG was recorded, using the standard three-electrode (10-20 system: parietal, occipital, and prefrontal) attachment. This signal and that of the standard three-electrode ECG were both processed by a bioelectric amplifier (Hewlett Packard 8811A) and recorded on the chart recorder and magnetic tape. Arterial oxygen saturation was measured in selected infants using a Biox IIA ear oximeter.

State classification. The sleep state for each minute was defined on the basis of the observed behavioral activity of the infant and its EEG record within that minute. The presence or absence of gross body movements, facial expressions, eye movements and vocalizations were recorded at 10 -s intervals on the chart during the study period. These observations were used to define the sleep state as either active sleep or quiet sleep using standard behavioral classification criteria (12-14). Although the presence of indeterminant sleep was recognized as a separate entity it occupied only a short portion of the monitoring period and was included in active sleep.

The EEG signal was classified as high voltage (quiet sleep) and low voltage (active sleep). Where EEG recordings were free of movement artifact, the correspondence between EEG and behavioral score was found to be $91 \%$.

Data analysis. Inasmuch as our aim was primarily to describe changes in temporal structure of periodic breathing with postnatal age, only those infants who had significant amounts of periodic breathing during at least one sleep recording were included in this analysis. This constraint reduced the study group to four infants. We performed 36 monitoring sessions of nominally 2 -h duration on these infants during the study period.

Data from each recording session were scanned manually to identify all occurrences (epochs) of periodic breathing defined as two or more sequential apneas of greater than 3-s duration, separated by periods of above-average ventilation with a duration of $\leq 20$ s. Only records in which the apneas occurred simultaneously on both the thermistor tracing and the abdominal tracing were considered for analysis. All the epochs identified were labeled as occurring in quiet or active sleep, and a mean PCD was determined for each epoch and then for each state. PCD was defined as the interval between the end of successive apneic periods (i.e. the time interval between the initial breath of one breathing/respiratory pause cycle and the initial breath of the next). This definition of PCD is similar to that used in our previous studies $(8,9)$. 
Where the epoch was longer than 25 cycles the epoch was considered to be a long epoch and average PCD and V/A ratios were calculated at half-minutely intervals over the complete epoch. Only epochs that were free of recording artifact were used, and this limited our choice in this part of the analysis to those occurring in quiet sleep.

Statistical analysis. Because the maturation of PCD with postnatal age was characterized by an initial segment where PCD fell followed by a segment where it was more or less constant, the change of PCD with postnatal age was described quantitatively using a bilinear regression. This regression technique fits two regression lines to the data, one on either side of a break point designated $\mathrm{PNA}_{\mathrm{BR}}$. The line for PNA $<\mathrm{PNA}_{\mathrm{BR}}$ is fitted to the total data set using a single slope linear regression model, but points for which $\mathrm{PNA} \geq \mathrm{PNA}_{\mathrm{BR}}$ are modified to $\mathrm{PNA}=\mathrm{PNA}_{\mathrm{BR}}$. The second line has zero slope and passes through the mean PCD for data $>\mathrm{PNA}_{\mathrm{BR}}$. $\mathrm{PNA}_{\mathrm{BR}}$ is adjusted iteratively to minimize the residual sum of the squared deviations from the bilinear prediction. This technique has been applied to the postnatal PCD data for the preterm infants plotted in Fig. 1. In Table 1 we have summarized an analysis of variance which shows that a significantly improved fit to the data is achieved by using this technique $\left(F_{1,26}=5.36 ; p<\right.$ $0.05)$ compared with a single regression line.

Unless otherwise stated figures quoted in the text are mean \pm SEM. Statistical comparisons were made using the $t$ test statistic with $p<0.05$ being used as the acceptance level.

\section{RESULTS}

$\boldsymbol{P C D}$. Because there was no significant difference in mean PCD between active and quiet sleep during any of the recordings made, the data were pooled so that a single mean PCD was

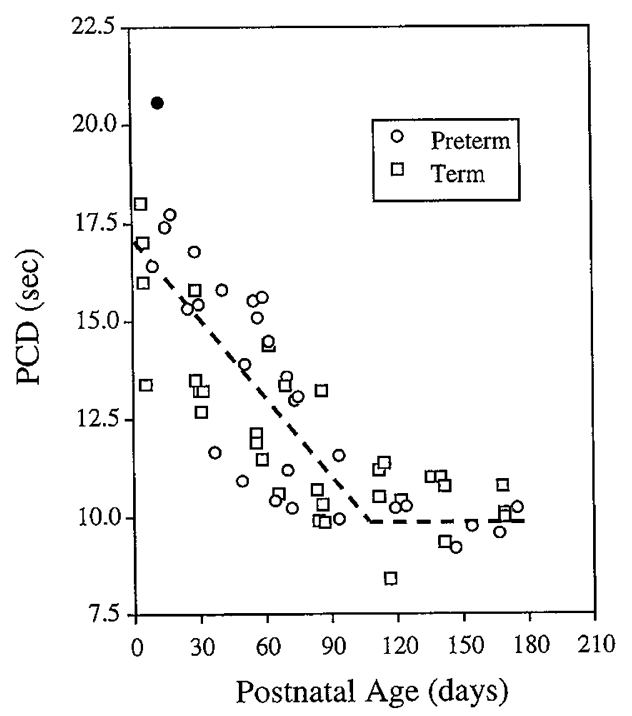

Figure 1. Combined $\mathrm{PCD}$ data for the term and preterm infants over a postnatal age range of birth to 6 mo of age showing the characteristic fall in PCD with postnatal age up to a break point of $105 \mathrm{~d}$ in the preterm infants (bilinear regression is shown as a broken line). A similar analysis gave a breakpoint of $90 \mathrm{~d}$ for the term infants. The regression slopes were not significantly different. The filled symbol - is a case of periodic obstructive sleep apnea in one preterm infant at PNA $=10 \mathrm{~d}$; notice the apparent prolongation of PCD in this case.
Table 1. Analysis of variance for bilinear regression

\begin{tabular}{lrrrrr}
\hline \multicolumn{1}{c}{ Source of variation } & $d f$ & SS & MSS & $F$ ratio & $F_{0.05}$ \\
\hline Residual for mean & 28 & 203.03 & & & \\
Single slope & 1 & 129.62 & 129.62 & & \\
& & & & 47.65 & 4.21 \\
Residual for single slope & 27 & 73.41 & 2.72 & & \\
Addition of second slope & 1 & 12.55 & 12.55 & & \\
& & & & 5.36 & 4.22 \\
$\begin{array}{l}\text { Residual for bilinear } \\
\quad \text { regression }\end{array}$ & 26 & 60.86 & 2.34 & & \\
\hline
\end{tabular}

$\mathrm{SS}=$ sum of Square; MSS = mean square.

available at each postnatal age for each infant. There was a significant decrease in mean PCD $(r=0.8)$ with increasing postnatal age (Fig. 1). The PCD fell from $17.1 \pm 3.3 \mathrm{~s}$ (mean $\pm 2 \mathrm{SD})$ at $9 \mathrm{~d}$ to $9.8 \pm 3.2 \mathrm{~s}($ mean $\pm 2 \mathrm{SD})$ at $105 \mathrm{~d}$ with a regression slope of $-0.072 \mathrm{~s} / \mathrm{d}$. There was no apparent change in PCD between $105 \mathrm{~d}$ and $6 \mathrm{mo}$. When the data for individual babies were examined for PNA $<105 \mathrm{~d}$, there was a highly significant fall in PCD for all infants; (mean $r=0.94$; range, $0.87-0.96)$. These results were similar to previous data (8) in term infants. In term infants, PCD fell from $15.6 \pm 2.7 \mathrm{~s}$ (mean $\pm 2 \mathrm{SD}$ ) at $1 \mathrm{wk}$ to $10.4 \pm 2.6 \mathrm{~s}($ mean $\pm 2 \mathrm{SD}$ ) at $90 \mathrm{~d}$. The regression slope was $-0.06 \mathrm{~s} / \mathrm{d}$ over this period. After $90 \mathrm{~d}$ there was no change in PCD up to 6 mo. There was no significant difference in regression slope between the term and preterm infants.

Long epoch analysis. At least one suitable long epoch was identified in each of the babies in the study and all of these occurred after a sigh during quiet sleep. Analysis was performed on a total of five epochs from the four infants with significant periodic breathing. All of these epochs displayed similar trends in the V/A ratio with time (see Fig. 2). There was a consistent fall in V/A ratio from the start of the epoch reaching a minimum value at a variable time designated $T_{\text {min }}$.
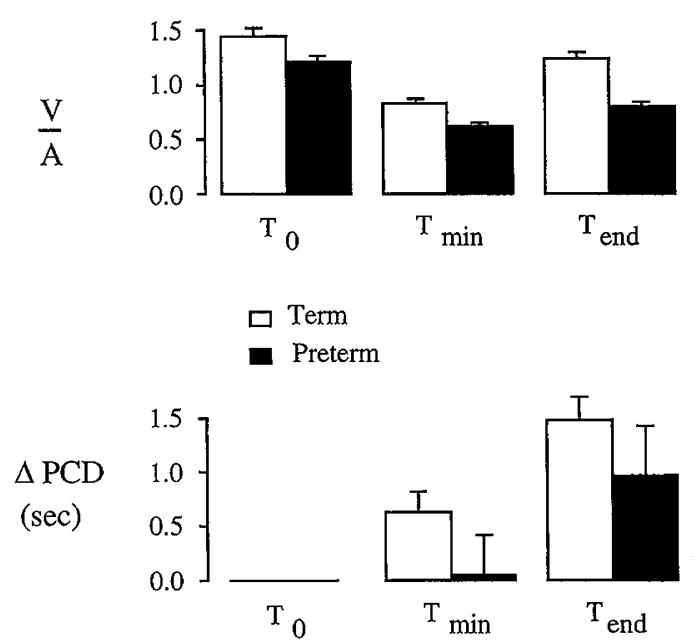

Figure 2. (Top panel) Mean V/A ratio during prolonged epochs of periodic breathing. The mean V/A ratio falls from the start of an epoch $\left(T_{\mathrm{o}}\right)$ until it reaches a minimum value at a time $T_{\min }$ and rises thereafter until the epoch terminates at $T_{\text {end }}$. (Bottom panel) PCD duration increases between $T_{\mathrm{o}}$ and $T_{\text {end }} . \triangle \mathrm{PCD}$ is defined as the difference in PCD at any time in the epoch with respect to that at $T_{\mathrm{o}}$. This characteristic change in PCD was found in both term and preterm infants. 
The total time from the start of the epoch to $T_{\min }$ varied from 5.0 to 19.0 min (mean $\pm \mathrm{SEM}=7.2 \pm 1.3 \mathrm{~min} ; n=5$ ). This compares with 5.0-18.0 min (mean $9.8 \pm 2.8 \mathrm{~min} ; n=5$ ) in the full-term infants (8). After $T_{\min }$ the V/A ratio rose again until the epoch ended, usually by a state change and/or an arousal from sleep. The values measured for the V/A ratio at $T_{0}, T_{\min }$, and $T_{\text {end }}$ were $1.21 \pm 0.08,0.62 \pm 0.03$, and $0.80 \pm$ 0.05 . The comparative data in the term infants were $1.45 \pm$ $0.11,0.83 \pm 0.02$, and $1.24 \pm 0.07$, respectively. The V/A ratio at $T_{\min }$ was significantly lower than at $T_{\mathrm{o}}(p<0.05, n=$ 5 ) in both term and preterm infants. In addition, the V/A ratio reached a significantly lower value in the preterm infants, 0.62 \pm 0.03 versus $0.83 \pm 0.02$ in the term infants $(p<0.05 ; n=$ 5).

During long epochs, the difference in PCD $(\triangle \mathrm{PCD})$ between the start of the epoch at $T_{\mathrm{o}}$ and the end at $T_{\min }$ was not significantly different from $0(p>0.05 ; n=5)$. Hence the fall in V/A ratio which occurred between $T_{\mathrm{o}}$ and $T_{\min }$ was mediated via an increase in the apnea duration (A) at the expense of the ventilation period $(V)$ with no change in PCD. For the changes in mean V/A ratio quoted the calculated percentage increase in apneic duration is $36 \%$.

There was a small but significant increase in PCD between $T_{\min }$ and $T_{\text {end }}$ similar to that found in the term infants. The mean increase at the end of the epoch $\left(T_{\text {end }}\right)$ was $0.96 \pm 0.46$ $\mathrm{s}$ (significantly greater than $0 ; p<0.05 ; n=5$ ). In the term infants the change was $1.48 \pm 0.21 \mathrm{~s}$ (significantly greater than $0 ; p<0.01 ; n=5$ ).

Where arterial oxygen saturation was measured during long epochs a consistent reduction in the mean $\mathrm{SaO}_{2}$ was measured which mirrored the fall in V/A ratio with time. As mean $\mathrm{SaO}_{2}$

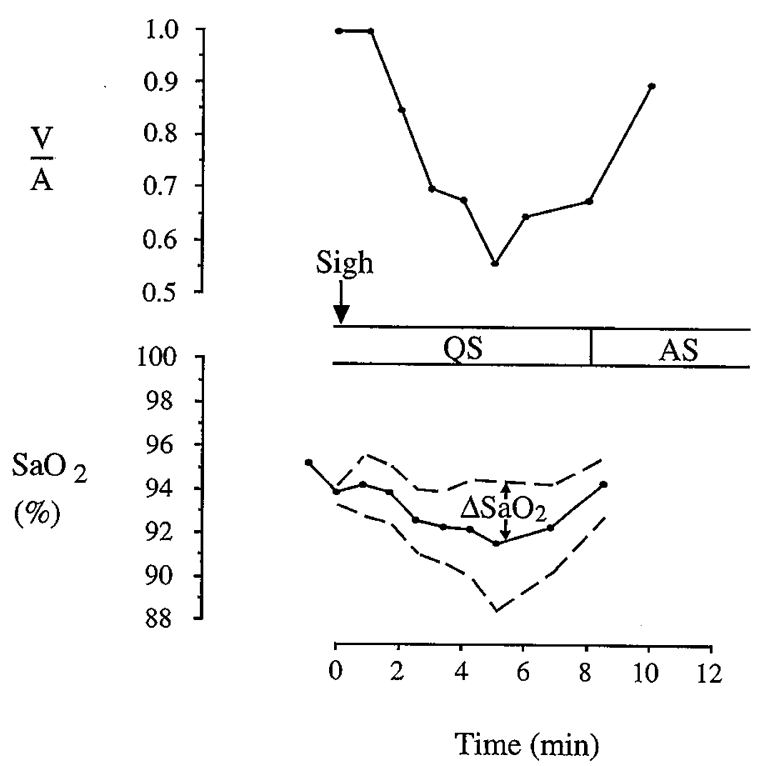

Figure 3. (Top panel) Typical recording of the progressive change in V/A ratio with time in one of our preterm infants during a long epoch of periodic breathing. Notice the characteristic fall in V/A after the sigh-induced onset. (Bottom panel) Also of note is the progressive fall in $\mathrm{SaO}_{2}$ (solid line) and the increasing amplitude $\left(\mathrm{SSaO}_{2}\right)$ of the oscillatory component of $\mathrm{SaO}_{2}$, associated with the alternating periods of apnea and respiratory activity which occur during periodic breathing. The maximum excursion of these swings is shown by broken lines. fell, the peak to trough swings in $\mathrm{SaO}_{2}\left(\Delta \mathrm{SaO}_{2}\right.$ in Fig. 3) associated with the respiratory oscillations of periodic breathing increased between $T_{\mathrm{o}}$ and $T_{\mathrm{min}}$ and then fell as V/A rose toward the end of the epoch. A typical result is shown in Figure 3.

\section{DISCUSSION}

The main findings of this study in relation to preterm infants classified as periodic breathers were the characteristic decrease in the oscillatory PCD of periodic breathing between $1 \mathrm{wk}$ and 3 mo with little change thereafter, and the systematic changes in the V/A ratio within long epochs of periodic breathing. These findings are similar to those we $(8,9)$ and others (7) have previously reported in term infants. Our data showing the decrease in PCD with maturation in preterm infants are clearly at variance with the study of Glotzbach et al. (15) which showed no significant change in PCD with postnatal age in a group of 51 preterm infants. However, there are several methodologic differences between the two studies that might explain the discrepancies between them.

First, our study was done during documented sleep (afternoon nap) after a feed whereas the Glotzbach et al. studies were performed over $24 \mathrm{~h}$ and only once on each baby in an intermediate or intensive care nursery setting just before discharge. The time when PCD was measured was not given and sleep state was not monitored. Only two long epochs per infant were selected for study whereas we used all epochs. Hence circadian effects on PCD, variability of PCD within and between infants, the effect of sleep disturbances, and the systematic changes in PCD during long epochs demonstrated in this study could be expected to inflate the variability in PCD measured and hence to contribute to the reported lack of change in PCD with postnatal age.

Second, and probably more importantly, the studies examined quite different age ranges. The oldest infants studied just before discharge from the nursery by Glotzbach et al. were 11 wk of age, whereas our infants were followed out to $25 \mathrm{wk}$. Seventy five percent of their data were clustered within the first 6 postnatal weeks (where even our data in Fig. 1 do not show a convincing trend) and only $8 \%$ is in the age range $10-12 \mathrm{wk}$. In our study, the period in which PCD ceased to vary with postnatal age did not occur until $105 \mathrm{~d}(15-16 \mathrm{wk})$, well beyond the end of their study. Furthermore, at $105 \mathrm{~d}$ and beyond both our study and that of Fleming et al. (7) have shown that long epochs are unlikely so that their study design, which required long epochs greater than 2 min in duration, may have precluded any measurement of PCD even if shorter epochs were present. Similarly they may also have precluded the early changes in PCD associated with the short epochs, before 1 wk, observed by Fleming et al. (7). Both of these omissions and the increased variability associated with their sampling method may have inadvertently biased their results so that the trend in PCD with postnatal age was obscured.

A final point which makes a comparative evaluation of their work and ours difficult is that an analysis of the data presented in their Figure 3, which relates PCD to postnatal age, reveals that the data are apparently not compatible with the mean appearing in their Table 1. Specifically, the mean PCD that we 
have calculated from their Figure 3 is $14.6 \mathrm{~s}$, whereas the mean PCD in their Table 1 is $15.2 \mathrm{~s}$. This suggests that the graph relating PCD and postnatal age is in error, because the data in the two other graphs in their Figure 3 are consistent with a mean of $15.2 \mathrm{~s}$.

The spontaneous decrease we have described in V/A ratio during long epochs of periodic breathing during quiet sleep is similar to the change reported by Rigatto and Brady (16) in infants challenged with hypoxia. Because hypoxia would increase peripheral chemoreflex sensitivity (i.e. $\mathrm{O}_{2}$ controller gain) (17), we hypothesize that increased controller gain leads to decreased V/A ratio. Rigatto and Brady (18) also reported one case of a progressive decrease in V/A ratio occurring in an infant who was periodic breathing while breathing air. It is quite apparent from their Figure 10 (18) that significant hypoxia (and hypercapnia) is developing during the period over which V/A is decreasing, suggesting that the periodic breathing observed is being stimulated by increased $\mathrm{O}_{2}$ controller gain secondary to hypoxemia. Furthermore, this epoch of periodic breathing ended in prolonged apnea, thus supporting the observations of Daily et al. (3).

Further support for these concepts comes from the servorespirator experiments of Cherniack et al. (19) in adult cats. In these experiments the animal's respiration was driven by an external ventilator controlled directly from the respiratory activity recorded from the cut end of the phrenic nerve. Changes in the relationship between neural drive and the tidal volume delivered by the ventilator (i.e. servo-ventilator gain) were controlled externally. They showed that increased servoventilator gain causes an increase in the duration of the apneic interval (A) at the expense of the duration of the hyperventilation phase, with the total cycle length remaining constant, i.e. V/A was reduced as "controller gain" was increased. This finding resembles our observations where V/A ratio fell during long epochs of periodic breathing with little change in PCD.

Hence the existing experimental evidence suggests that the decrease in V/A ratio which we observed during long epochs of periodic breathing could be attributed to an increase in respiratory controller gain as the epoch progresses. This increase in gain could be explained by the progressive hypoxemia which develops in association with hypoventilation secondary to central respiratory depression (18). Our preliminary data on arterial oxygen saturation showing a progressive desaturation during the early stages of the epoch and an increased oscillatory amplitude (Fig. 3) also support this view. Hence, the existing evidence strongly indicates that the chemical control system plays a key role in the establishment of respiratory instabilities, and in determining the duration of the cycling periods of ventilation and apnea during unstable breathing periods, in both preterm and term infants, as well as in adult animals.

Just as observed changes in the V/A ratio can be explained on the basis of known features of the chemical control system, variation in the PCD of periodic breathing might also be explained from the standpoint of chemical control. However, at the moment we are limited in the conclusions we can draw, because we do not have values for many of the physiologic variables needed to obtain a clear view as to whether chemical control mechanisms underlie the postnatal decline in this vari- able. Nevertheless, it is important to pursue the point, because PCD declines to its minimum value across an age range that encompasses the peak incidence of SIDS, and an understanding of the factors involved may help us to understand what makes the infant respiratory control system susceptible to failure.

The fall in oscillatory PCD with postnatal age that we have identified in both preterm and term infants was unexpected on the basis of predictions made using the general theoretical model of periodic breathing proposed by Khoo et al. (10). They used allometric scaling of an adult model to predict PCDs of 18 $\mathrm{s}$ for preterm infants. Although this is in good agreement with our data and that of others $(18,20)$, application of the scaling relationship predicts that $\mathrm{PCD}$ would increase with age, because PCD and body weight (BW) are related by a power function $\left(\mathrm{PCD} \alpha \mathrm{BW}^{0.25}\right)$. Thus, neither our study nor the study of Glotzbach et al. lends support to these theoretical predictions.

The factors which promote periodic breathing have been discussed by a number of authors $(8-10)$. Briefly three factors can be identified: 1 ) the extension of the delay time between the lung and the peripheral chemoreceptors (this probably includes both peripheral receptor latency and circulatory delays), 2) reduced lung gas stores that cause reduced damping, and 3) exponentially increased respiratory drive from the peripheral receptors due to the mild hypoxemia associated, for example, with sigh induced apnea or reduced central respiratory drive. All of these factors can contribute to ventilatory overshoot in response to a disturbance to ventilation and hence can cause periodic breathing.

Factors which can change PCD are less well understood. Clearly any factor which can accelerate the changes in blood gases which occur at the carotid chemoreceptors in response to ventilatory disturbances will ultimately reduce PCD. Theoretically Khoo et al. (10) showed that the most important factors include circulation delays from the lung to carotid chemoreceptor and the lung washout times by ventilation and perfusion. In a developmental setting, receptor latency, maturation of neural respiratory pathways, and the stability of the apneic threshold for $\mathrm{O}_{2}$ and $\mathrm{CO}_{2}$ might also be important and may even dominate these trends.

Bolton (21) has shown that the lung exchange time (analogous to the lung washout by ventilation) is extended in neonates compared with adults, but no data were presented on how this time changed postnatally. We are unaware of data on lung washout time by perfusion or as to whether this changes with postnatal age.

Similarly, there are no data on total body circulation delay times over the first months of life, but maturational data in children and adults (22) indicate that these increase with age above $1 \mathrm{y}$. Theoretically, such an increase, if it can be extrapolated to the under 1-y age range, is likely to extend PCD with advancing postnatal age, not shorten it as we have observed.

A maturational increase in sensitivity of the carotid body to hypoxia (and hypercapnia) could also contribute to the change in PCD. Such an increase has been implied in newborn lambs by Mayock et al. (23) who showed that exogenous dopamine inhibited carotid body function more at 2 wk than at birth. 
These results suggest that a maturational increase in the sensitivity of the carotid body does occur postnatally. However, experiments in which the reflex sensitivity of the respiratory controller are artificially changed (19) indicate that the PCD is relatively independent of such changes, even though the V/A ratio decreases (see V/A discussion earlier). Hence increases in carotid body sensitivity with age probably do not explain the observed changes in PCD.

Change in latency with postnatal age of the peripheral receptor response to hypoxemia has been mentioned previously $(8,9)$ as a factor which could reduce PCD. There are no data on the maturation of receptor response in human neonates, but Belenky et al. (24) reported that the response time to hypoxia in newborn lambs compared at birth and 2 wk postnatally fell from 5.7 to $1.8 \mathrm{~s}$. They suggested that the development of a rapid carotid body-mediated response to hypoxia occurred gradually during the first days of postnatal life. This reduction might explain, at least in part, the reduction in PCD we have observed.

Clearly, an exact analysis of the crucial factor or factors which lead to a reduction in PCD with postnatal age cannot be made at present because a complete quantitative data set documenting changes in circulation delay, chemoreceptor sensitivity, lung washout time by perfusion, and chemoreceptor latency is not available for the human infant. It would appear that studies aimed at quantifying the changes in lung washout time and chemoreceptor latency might be fruitful in elucidating the cause of the observed reduction.

We conclude that important maturational changes occur in the respiratory control system of the premature infant over the first 6 mo postnatally and these changes are reflected in a reduction in the $\mathrm{PCD}$ of periodic breathing over this same period. These findings are similar to those previously demonstrated in term infants (7-9). In addition, the characteristic changes in respiratory pattern which occur during long epochs of periodic breathing are consistent with a role for chemical stimuli in the genesis of periodic breathing.

Acknowledgments. The authors thank Fiona Wilson and Anthony Strangward for their assistance with data analysis and Christine Jones for assistance in the preparation of the manuscript.

\section{REFERENCES}

1. Glotzbach SF, Baldwin RB, Lederer BA, Tansey PA, Ariagno RL 1989 Periodic breathing in preterm infants: incidence and characteristics. Pediatrics 84:785-792
2. Glotzbach SF, Ariagno RL 1992 Periodic Breathing. In: Beckerman RC, Brouillette RT, Hunt CE (eds) Respiratory Control Disorders in Infants and Children. Williams \& Wilkins, Baltimore, pp 142-160

3. Daily WJR, Klaus M, Meyer HBP 1969 Apnea in premature infants: monitoring, incidence, heart rate changes, and an effect of environmental temperature. Pediatrics 43:510-518

4. Barrington KJ, Finer NN 1990 Periodic breathing and apnea in preterm infants. Pediatr Res 27:118-121

5. Kelly DH, Shannon DC 1979 Periodic breathing in infants with near miss sudden infant death syndrome. Pediatrics 63:355-360

6. Kelly DH, Walker AM, Cahen L, Shannon DC 1980 Periodic breathing in siblings of sudden infant death syndrome victims. Pediatrics 66:515-520

7. Fleming PJ, Goncalves AL, Levine MR, Woollard S 1984 The development of stability of respiration in human infants. Changes in ventilatory responses to spontaneous sighs. J Physiol 347:1-6

8. Wilkinson MH, Cranage SC, Adamson TM, Wilson FE, Strangward A, Walker AM 1986 Incidence and temporal structure of oscillatory breathing patterns in full term neonates. Proceedings of the IEEE Engineering in Medicine and Biolology, 8th Annual Conference, Dallas-Fort Worth, Texas, Vol. 2, pp 1257-1262

9. Barrington K, Finer N, Wilkinson MH 1987 Progressive shortening of periodic breathing cycle duration in normal infants: an index of peripheral chemoreceptor maturation. Pediatr Res 21:247

10. Khoo MC, Kronauer RC, Strohl KP, Slutsky AS 1982 Factors inducing periodic breathing in humans: a general model. J Appl Physiol Respir Environ Exercise Physiol 53:644-659

11. Adamson TM, Cranage S, Maloney JE, Wilkinson MH, Wilson FE, Yu VYH 1981 The maturation of respiratory patterns in normal full-term infants during the first six postnatal months. I. Sleep states and respiratory variability. Aust Paediatr J 17:250256

12. Wolff PH 1971 Organisation of behaviour in the first three months of life. In: Nurberger JI (ed) Biological and Environmental Determinants of Early Development. Williams \& Wilkins, Baltimore

13. Prechtl HF, Weinmann H, Aikyama Y Organisation of physiological parameters in normal and neurologically abnormal infants. Neuropaediatrie 1:101-129, 1969

14. Thoman EB 1975 Early development of sleeping behaviour in infants. In: Ellis N (ed) Aberrant Development in Infancy: Human and Animal Studies. Halsted Press, New York

15. Glotzbach SF, Tansey PA, Baldwin RB, Ariagno RL 1989 Periodic breathing cycle duration in preterm infants. Pediatr Res 25:258-261

16. Rigatto H, Brady JP 1972 Periodic breathing and apnea in preterm infants. II. Hypoxia as the primary event. Pediatrics 50:219-228

17. Cherniack NS, vonEuler C, Homma I, Kao FF 1978 Effects of increased respiratory controller gain during hypoxia and hypercapnia on periodic breathing in cats. Adv Exp Med Biol 99:423-431

18. Rigatto H, Brady JP 1972 Periodic breathing and apnea in preterm infants. I. Evidence for hypoventilation possibly due to central respiratory depression. J Pediatr 50:202218

19. Cherniack NS, Von Euler C, Homma I, Kao FF 1979 Experimentally induced Cheyne-Stokes breathing. Respir Physiol 37:185-200

20. Waggener TB, Frantz ID, Stark AR, Kronauer RE 1982 Oscillatory breathing patterns leading to apneic spells in infants. J Appl Physiol Respir Environ Exercise Physiol 52:1288-1295

21. Bolton DPG 1979 Diffusional Inhomogeneity: gas mixing efficiency in the new-born lung. J Physiol 286:447-455

22. Blumgart HL, Rowlands S 1971 Circulation time: vertebrates. Biol Handb 417-424

23. Mayock DE, Standaert TA, Guthrie RD, Woodrum DE 1983 Dopamine and carotid body function in the newborn lamb. J Appl Physiol Respir Environ Exercise Physiol $54: 814-820$

24. Belenky DA, Standaert TA, Woodrum DE 1979 Maturation of ventilatory response of the newborn lamb. J Appl Physiol Respir Environ Exercise Physiol 47:927-930 